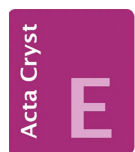

CRYSTALLOGRAPHIC COMMUNICATIONS

ISSN 2056-9890

Received 16 December 2019

Accepted 14 January 2020

Edited by K. Fejfarova, Institute of Biotechnology CAS, Czech Republic

Keywords: crystal structure; stannylene; $\eta$-2 coordination; indenyl donor group.

Supporting information: this article has supporting information at journals.iucr.org/e

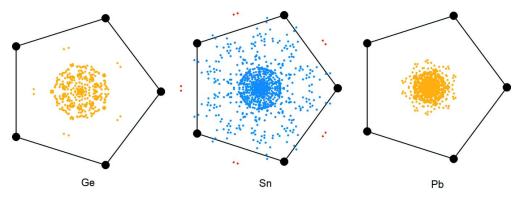

OPEN Ә ACCESS

\section{An indenide-tethered N-heterocyclic stannylene}

\author{
Tobias Bischof, Kieren J. Evans, Mairi F. Haddow* and Stephen M. Mansell*
}

Institute of Chemical Sciences, School of Engineering and Physical Sciences, Heriot-Watt University, Edinburgh, EH14 4AS, Scotland. *Correspondence e-mail: M.Haddow@hw.ac.uk, S.Mansell@hw.ac.uk

The structure of $\left(\mu-1 \kappa N: 2\left(\eta^{2}\right), \kappa^{2} N, N^{\prime}-(2-\{[2,6\right.$-bis(propan-2-yl)phenyl]azanidyl\}ethyl)[2-(1H-inden-1-yl)ethyl]azanido $)(1,4,7,10,13,16$-hexaoxacyclooctadecane-1 $\left.\kappa^{6} O\right)$ lithiumtin, $\left[\mathrm{LiSn}\left(\mathrm{C}_{8} \mathrm{H}_{16} \mathrm{O}_{4}\right)\left(\mathrm{C}_{25} \mathrm{H}_{31} \mathrm{~N}_{2}\right)\right]$, at $100 \mathrm{~K}$ has monoclinic $\left(P 2_{1} / n\right)$ symmetry. Analysis of the coordination of the $\mathrm{Sn}$ to the indenyl ring shows that the $\mathrm{Sn}$ interacts in an $\eta^{2}$ fashion. A database survey showed that whilst this coordination mode is unusual for $\mathrm{Ge}$ and $\mathrm{Pb}$ compounds, Sn displays a wider range of coordination modes to cyclopentadienyl ligands and their derivatives.

\section{Chemical context}

N-heterocyclic stannylenes (NHSns) are the tin analogues of $\mathrm{N}$-heterocyclic carbenes (NHCs). With an unsaturated backbone, they have been found to be thermally unstable (GansEichler et al., 2002, Gans-Eichler et al. 2006), but with a saturated backbone they are thermally robust (Mansell et al., 2008) and show interesting binding properties including a higher propensity for bridging coordination modes (Mansell et al., 2011).
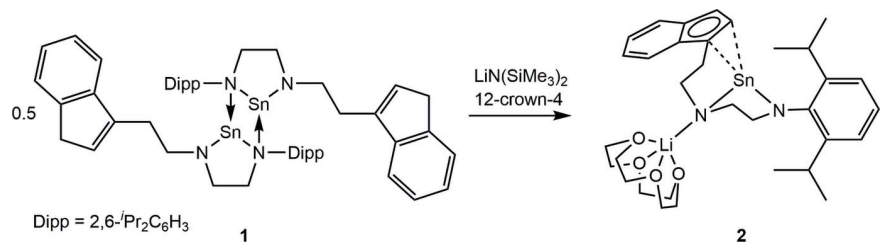

We have sought to install NHSns into a tethered ligand system using a fluorenyl group linked to the NHSn with a $\mathrm{C}_{2} \mathrm{H}_{4}$ linker, but this resulted in dimeric species with $\mathrm{Sn}-\mathrm{N}$ dative bonding, even upon addition of suitable $\mathrm{Rh}$ salts (Roselló-Merino \& Mansell, 2016). In this contribution we analyse the crystal structure of a monomeric NHSn with an indenyl donor group.

\section{Structural commentary}

The crystal structure of the title compound $\mathbf{2}$ shows a deprotonated indenide moiety connected to a diamidostannylene unit via a $\mathrm{C}_{2} \mathrm{H}_{4}$ linker. The lithium cation is bound to the less sterically hindered $\mathrm{N}$ atom $[\mathrm{Li}-\mathrm{N}=2.043(7) \AA]$, as well as to the 12-crown-4 tetradentate ether ligand (Fig. 1). The Sn atom is bonded to two $\mathrm{N}$ atoms [ $\mathrm{Sn}-\mathrm{N}=2.157$ (3) and 2.089 (3) $\AA$ ] and there appears to be an $\eta^{2}$ interaction with the indenyl anion $[\mathrm{Sn} \cdots \mathrm{C}=2.734(3)$ and $2.701(3) \AA]$ with $\mathrm{Sn} \cdots \mathrm{C}$ distances that are similar to those in stannocene $\left[\mathrm{Sn}\left(\eta^{5}-\mathrm{Cp}\right)_{2}\right]$,

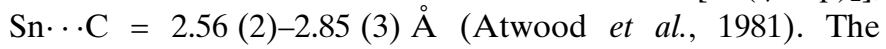
formation of $\mathbf{2}$ shows that the soft NHSn lone pair does not 


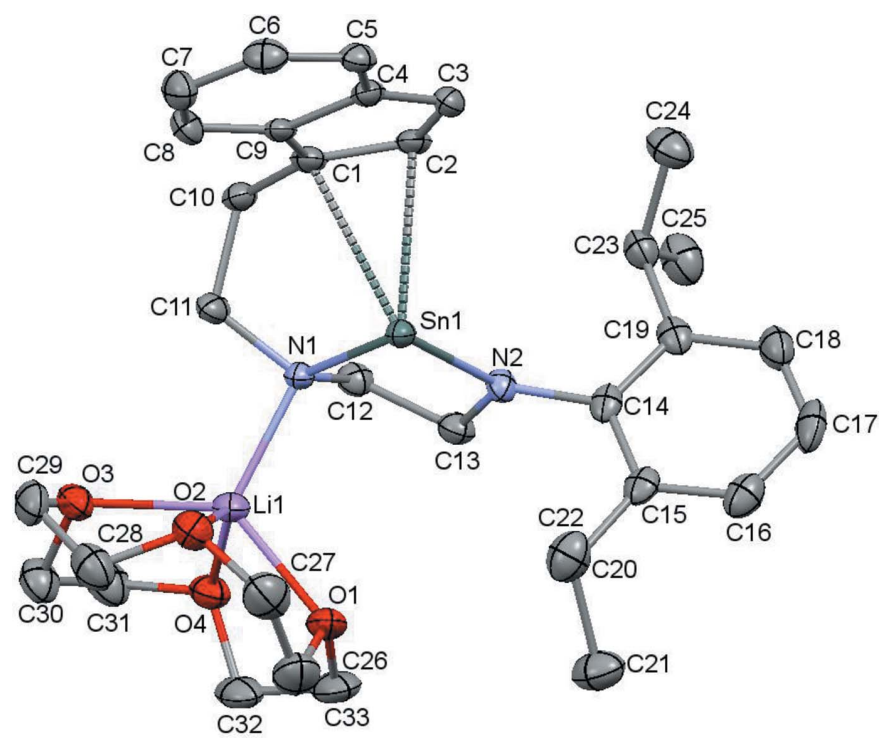

Figure 1

Displacement ellipsoid plot of $\mathbf{2}$ (shown at the $50 \%$ probability level) with all $\mathrm{H}$ atoms removed for clarity.

interact with the relatively hard $\mathrm{Li}$ cation, unlike the situation in the lithium complexes of tethered NHCs previously published (Evans \& Mansell, 2019; Evans et al., 2019).

\section{Database survey}

For the structure of $\mathbf{2}$, two $\mathrm{Sn}$...C distances are much shorter [2.734 (3) and $2.701(3) \AA]$ than the other three [3.193 (3), 3.222 (3) and 3.486 (3) $\AA$ ] in the five-membered ring of the indenyl moiety. The only two other crystallographically characterized Sn-indenyl complexes [ $\left.\mathrm{Sn}\left\{1,3-\left(\mathrm{SiMe}_{3}\right)_{2} \mathrm{C}_{9} \mathrm{H}_{5}\right\}_{2}\right]$ and $\left[\mathrm{Sn}\left(\mathrm{C}_{5} \mathrm{Me}_{5}\right)\left\{1,3-\left(\mathrm{SiMe}_{3}\right)_{2} \mathrm{C}_{9} \mathrm{H}_{5}\right\}\right]$ (Jones \& Cowley, 2005), have much less pronounced differences in the shortest and longest bond lengths [maximum range of $0.26 \AA$ compared to 2 , which has a range of $0.785 \AA$ ] although the bond lengths to two carbon atoms in the ring are shorter than the remaining three, which always includes the two benzannulated carbon atoms. This has been termed $\eta^{3}+\eta^{2}$ coordination (Calhorda \& Veiros, 1999).

By surveying the coordination of cyclopentadienyl ligands to main group atoms using the CSD (Version 5.40, update of August 2019; Groom et al., 2016), we can clearly see the flexible coordination modes of tin compared to other group 14 metals. The position of the metal was projected onto the plane of the $\mathrm{Cp}$ ring and these datapoints were expanded according to $C_{5 \mathrm{v}}$ symmetry (i.e. there are ten symmetry-equivalent data points for each crystal structure). The results are shown in Fig. $2 a-c$ for germanium, tin and lead, respectively. Germanium and lead are almost always projected near the centre of the $\mathrm{Cp}$ ring; however, tin shows a wide range of projection points. The datapoints for this structure are displayed in red in Fig. $2 b$, showing the distinct interaction with two carbon centres, a unique coordination mode for group 14 metals.

\section{Synthesis and crystallization}

\section{Synthesis of $\left[\mathrm{Sn}\left\{\left(\mathrm{N}, \mathrm{N}^{\prime}-\kappa^{2}-\left(\mathrm{C}_{9} \mathrm{H}_{7}\right) \mathrm{C}_{2} \mathrm{H}_{4} \mathrm{NC}_{2} \mathrm{H}_{4} \mathrm{~N}\left(2,6{ }^{i}{ }^{i} \mathrm{Pr}_{2^{-}}\right.\right.\right.\right.$ $\left.\left.\left.\mathrm{C}_{6} \mathrm{H}_{3}\right)\right\}\right]_{2}, 1$}

To a solution of $\left(\mathrm{C}_{9} \mathrm{H}_{7}\right) \mathrm{C}_{2} \mathrm{H}_{4} \mathrm{~N}(\mathrm{H}) \mathrm{C}_{2} \mathrm{H}_{4} \mathrm{~N}(\mathrm{H})\left(2,6-{ }^{i} \mathrm{Pr}_{2} \mathrm{C}_{6} \mathrm{H}_{3}\right)$ (Roselló-Merino \& Mansell, 2016) (330 mg, $0.91 \mathrm{mmol}$ ) in THF (5 ml), $\mathrm{Sn}\left[\mathrm{N}\left(\mathrm{SiMe}_{3}\right)_{2}\right]_{2}(400 \mathrm{mg}, 0.91 \mathrm{mmol})$ dissolved in THF $(2 \mathrm{ml})$ was added slowly at room temperature under nitrogen in a two-necked-flask in a glovebox. After $2 \mathrm{~h}$, the solvent was removed by pipette and the precipitate was washed five times with $5 \mathrm{ml}$ of petroleum ether by dispersing it and pipetting off the solvent after the residue had settled. Evaporation of the remaining solvent under high vacuum yielded the desired product as a light-yellow solid $(348 \mathrm{mg}$, $0.73 \mathrm{mmol}, 80 \%)$.

${ }^{1} \mathrm{H}$ NMR (400 MHz, $298 \mathrm{~K}, d^{8}$-THF): $\delta=7.5-6.9(m, \mathrm{Ar}-\mathrm{H})$, $6.28(\mathrm{~m}), 3.67(\mathrm{~d}), 3.48(\mathrm{~m}), 3.38(\mathrm{~m}), 3.00(\mathrm{~m}), 2.88(\mathrm{~m}), 2.79$ $(m), 1.19(d) ;{ }^{119} \mathrm{Sn}\left(149 \mathrm{MHz}, 298 \mathrm{~K}, d^{8}\right.$-THF); $\delta=79.7 \mathrm{ppm}$. Analysis calculated for $\mathrm{C}_{25} \mathrm{H}_{32} \mathrm{~N}_{2} \mathrm{Sn}$ : C 62.65, H 6.73, N 5.85; Found: C 62.53, H 6.66, N 5.68

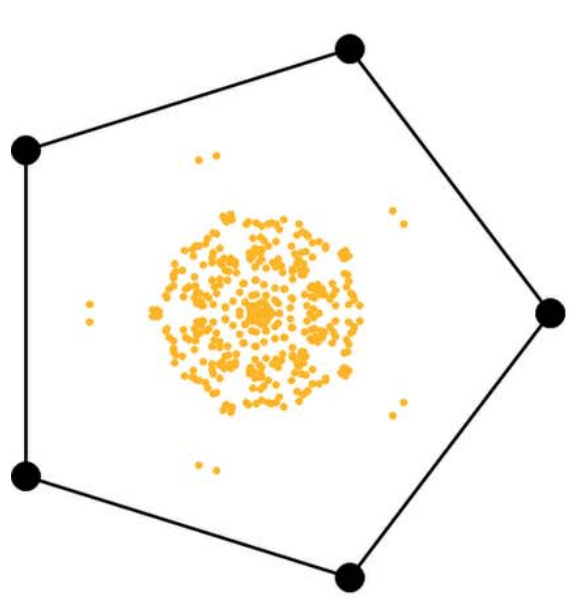

(a) $\mathrm{Ge}$

Figure 2

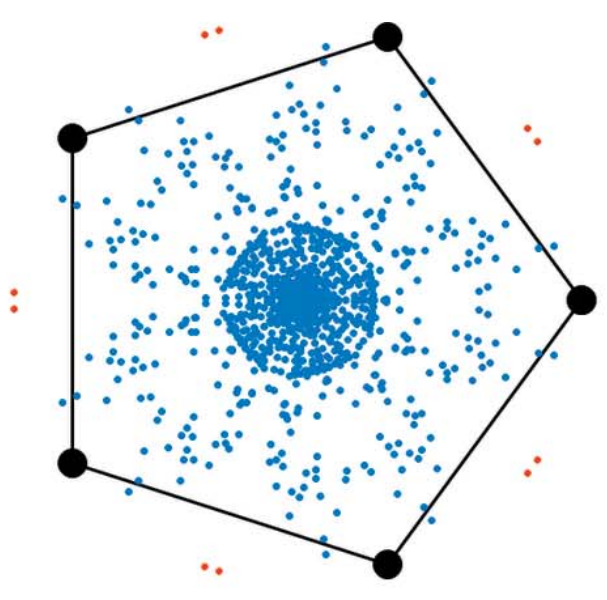

(b) $\mathrm{Sn}$

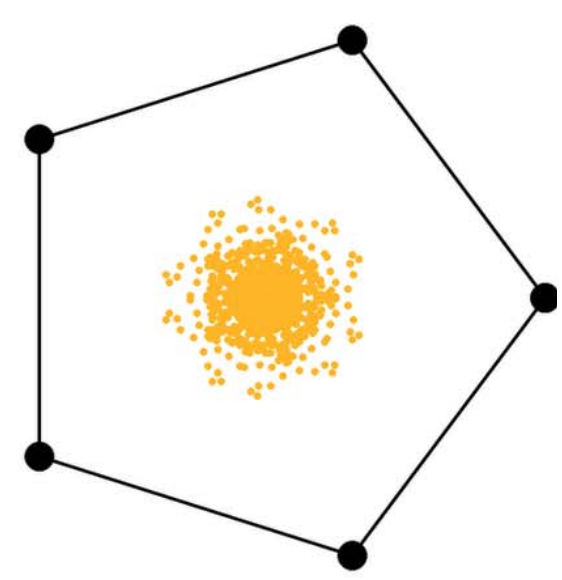

(c) $\mathrm{Pb}$

Projection plots of metal position onto idealized $\mathrm{Cp}$ ring. 
Synthesis of indenide-tethered $\mathbf{N}$-heterocyclic stannylene 2

To 1 (10 mg, $0.03 \mathrm{mmol})$ in a glass vial under nitrogen in a glovebox was added $\mathrm{Li}\left[\mathrm{N}\left(\mathrm{SiMe}_{3}\right)_{2}\right](5 \mathrm{mg}, 0.03 \mathrm{mmol})$ in THF $(0.5 \mathrm{~mL})$ then 12 -crown-4 $(11 \mathrm{mg}, 0.6 \mathrm{mmol})$ in THF $(0.2 \mathrm{ml})$. This vial was placed in a freezer, producing a small number of single crystals. Reactions on larger scales led to concentrations that were too high, leading to decomposition processes. The material that was produced was not soluble in $d^{8}$-THF.

\section{Refinement}

Crystal data, data collection and structure refinement details are summarized in Table $1 . \mathrm{H}$ atoms were positioned geometrically $(\mathrm{C}-\mathrm{H}=095-1.00 \AA)$ and refined using a riding model with $U_{\text {iso }}(\mathrm{H})=1.2 U_{\text {eq }}(\mathrm{C})$ or $1.5 U_{\text {eq }}(\mathrm{C}$-methyl $)$.

\section{Funding information}

Funding for this research was provided by: Engineering and Physical Sciences Research Council (PhD scholarship to K. J. Evans); Daphne Jackson Trust (award to M. F. Haddow).

\section{References}

Atwood, J. L., Hunter, W. E., Cowley, A. H., Jones, R. A. \& Stewart, C. A. (1981). J. Chem. Soc. Chem. Commun. pp. 925-927.

Bruker (2015). APEX2, SAINT and $S A D A B S$. Bruker AXS Inc., Madison, Wisconsin, USA.

Calhorda, M. J. \& Veiros, L. F. (1999). Coord. Chem. Rev. 185-186, 37-51.

Dolomanov, O. V., Bourhis, L. J., Gildea, R. J., Howard, J. A. K. \& Puschmann, H. (2009). J. Appl. Cryst. 42, 339-341.

Evans, K. J., Campbell, C. L., Haddow, M. F., Luz, C., Morton, P. A. \& Mansell, S. M. (2019). Eur. J. Inorg. Chem. pp. 4894-4901.

Evans, K. J. \& Mansell, S. M. (2019). Chem. Eur. J. 25, 37663769.

Gans-Eichler, T., Gudat, D., Nättinen, K. \& Nieger, M. (2006). Chem. Eur. J. 12, 1162-1173.

Gans-Eichler, T., Gudat, D. \& Nieger, M. (2002). Angew. Chem. Int. Ed. 41, 1888-1891.

Groom, C. R., Bruno, I. J., Lightfoot, M. P. \& Ward, S. C. (2016). Acta Cryst. B72, 171-179.
Table 1

Experimental details.

\begin{tabular}{|c|c|}
\hline \multicolumn{2}{|l|}{ Crystal data } \\
\hline Chemical formula & {$\left[\mathrm{LiSn}\left(\mathrm{C}_{8} \mathrm{H}_{16} \mathrm{O}_{4}\right)\left(\mathrm{C}_{25} \mathrm{H}_{32} \mathrm{~N}_{2}\right)\right]$} \\
\hline$M_{\mathrm{r}}$ & 661.35 \\
\hline Crystal system, space group & Monoclinic, $P 2_{1} / n$ \\
\hline Temperature $(\mathrm{K})$ & 100 \\
\hline$a, b, c(\AA)$ & $\begin{array}{l}9.9766(8), 17.7991(14), \\
\quad 18.8402(14)\end{array}$ \\
\hline$\beta\left(^{\circ}\right)$ & $95.510(4)$ \\
\hline$V\left(\AA^{3}\right)$ & $3330.1(4)$ \\
\hline$Z$ & 4 \\
\hline Radiation type & Мо $K \alpha$ \\
\hline$\mu\left(\mathrm{mm}^{-1}\right)$ & 0.80 \\
\hline Crystal size $(\mathrm{mm})$ & $0.20 \times 0.20 \times 0.08$ \\
\hline \multicolumn{2}{|l|}{ Data collection } \\
\hline Diffractometer & Bruker APEXII CCD \\
\hline Absorption correction & $\begin{array}{l}\text { Multi-scan (SADABS; Bruker, } \\
\text { 2015) }\end{array}$ \\
\hline$T_{\min }, T_{\max }$ & $0.614,0.746$ \\
\hline $\begin{array}{l}\text { No. of measured, independent and } \\
\text { observed }[I>2 \sigma(I)] \text { reflections }\end{array}$ & $25958,7615,5184$ \\
\hline$R_{\text {int }}$ & 0.051 \\
\hline$(\sin \theta / \lambda)_{\max }\left(\AA^{-1}\right)$ & 0.650 \\
\hline \multicolumn{2}{|l|}{ Refinement } \\
\hline$R\left[F^{2}>2 \sigma\left(F^{2}\right)\right], w R\left(F^{2}\right), S$ & $0.042,0.096,1.04$ \\
\hline No. of reflections & 7615 \\
\hline No. of parameters & 374 \\
\hline $\mathrm{H}$-atom treatment & $\mathrm{H}$-atom parameters constrained \\
\hline$\Delta \rho_{\max }, \Delta \rho_{\min }\left(\mathrm{e} \AA^{-3}\right)$ & $1.36,-0.59$ \\
\hline
\end{tabular}

Computer programs: APEX2 and SAINT (Bruker, 2013), SHELXT (Sheldrick, 2015a), SHELXL (Sheldrick, 2015b) and OLEX2 (Dolomanov et al., 2009).

Jones, J. N. \& Cowley, A. H. (2005). Chem. Commun. pp. 13001302.

Mansell, S. M., Herber, R. H., Nowik, I., Ross, D. H., Russell, C. A. \& Wass, D. F. (2011). Inorg. Chem. 50, 2252-2263.

Mansell, S. M., Russell, C. A. \& Wass, D. F. (2008). Inorg. Chem. 47, 11367-11375.

Roselló-Merino, M. \& Mansell, S. M. (2016). Dalton Trans. 45, 62826293.

Sheldrick, G. M. (2015a). Acta Cryst. A71, 3-8.

Sheldrick, G. M. (2015b). Acta Cryst. C71, 3-8. 


\section{Special details}

Geometry. All esds (except the esd in the dihedral angle between two 1.s. planes) are estimated using the full covariance matrix. The cell esds are taken into account individually in the estimation of esds in distances, angles and torsion angles; correlations between esds in cell parameters are only used when they are defined by crystal symmetry. An approximate (isotropic) treatment of cell esds is used for estimating esds involving l.s. planes.

Fractional atomic coordinates and isotropic or equivalent isotropic displacement parameters $\left(\hat{A}^{2}\right)$

\begin{tabular}{|c|c|c|c|c|}
\hline & $x$ & $y$ & $z$ & $U_{\text {iso }} * / U_{\text {eq }}$ \\
\hline Sn1 & $0.62831(2)$ & $0.62730(2)$ & $0.73575(2)$ & $0.01853(8)$ \\
\hline $\mathrm{O} 1$ & $0.7755(3)$ & 0.69869 (14) & $0.93346(13)$ & $0.0333(6)$ \\
\hline $\mathrm{O} 2$ & $0.9148(3)$ & 0.75607 (14) & $0.82933(13)$ & $0.0337(6)$ \\
\hline $\mathrm{O} 3$ & $0.7698(3)$ & $0.88333(13)$ & $0.83378(12)$ & $0.0291(6)$ \\
\hline $\mathrm{O} 4$ & $0.6260(3)$ & 0.82577 (14) & $0.93572(12)$ & $0.0311(6)$ \\
\hline N1 & $0.5656(3)$ & $0.73222(15)$ & $0.77937(13)$ & $0.0189(6)$ \\
\hline N2 & $0.4936(3)$ & $0.58853(15)$ & 0.80578 (14) & $0.0208(6)$ \\
\hline $\mathrm{C} 1$ & 0.5055 (4) & $0.70370(19)$ & $0.62151(17)$ & $0.0221(8)$ \\
\hline $\mathrm{C} 2$ & 0.4399 (3) & $0.63288(19)$ & $0.62289(16)$ & $0.0215(7)$ \\
\hline $\mathrm{H} 2$ & 0.3654 & 0.6220 & 0.6490 & $0.026^{*}$ \\
\hline $\mathrm{C} 3$ & 0.5018 & $0.58133(19)$ & 0.57967 (17) & $0.0226(8)$ \\
\hline H3 & 0.4765 & 0.5303 & 0.5718 & $0.027^{*}$ \\
\hline $\mathrm{C} 4$ & $0.6080(3)$ & $0.61883(18)$ & $0.55015(16)$ & $0.0202(7)$ \\
\hline $\mathrm{C} 5$ & 0.7031 (4) & $0.5971(2)$ & $0.50330(17)$ & $0.0233(8)$ \\
\hline H5 & 0.7044 & 0.5468 & 0.4865 & $0.028^{*}$ \\
\hline C6 & 0.7938 (4) & $0.6485(2)$ & $0.48200(19)$ & $0.0293(9)$ \\
\hline H6 & 0.8570 & 0.6335 & 0.4501 & $0.035^{*}$ \\
\hline C7 & 0.7942 (4) & $0.7236(2)$ & $0.50699(19)$ & $0.0303(9)$ \\
\hline H7 & 0.8569 & 0.7587 & 0.4913 & $0.036^{*}$ \\
\hline $\mathrm{C} 8$ & $0.7050(4)$ & $0.7461(2)$ & $0.55352(18)$ & $0.0264(8)$ \\
\hline H8 & 0.7068 & 0.7965 & 0.5703 & $0.032 *$ \\
\hline $\mathrm{C} 9$ & $0.6111(4)$ & $0.69545(18)$ & $0.57669(17)$ & $0.0198(8)$ \\
\hline $\mathrm{C} 10$ & $0.4676(4)$ & $0.77556(19)$ & $0.65700(17)$ & $0.0235(8)$ \\
\hline $\mathrm{H} 10 \mathrm{~A}$ & 0.3728 & 0.7720 & 0.6681 & $0.028^{*}$ \\
\hline H10B & 0.4738 & 0.8177 & 0.6232 & $0.028^{*}$ \\
\hline C11 & 0.5574 (4) & $0.79301(18)$ & $0.72628(17)$ & $0.0237(8)$ \\
\hline H11A & 0.6495 & 0.8044 & 0.7139 & $0.028^{*}$ \\
\hline H11B & 0.5224 & 0.8387 & 0.7482 & $0.028^{*}$ \\
\hline C12 & $0.4363(4)$ & 0.72044 (19) & $0.80929(18)$ & $0.0231(8)$ \\
\hline $\mathrm{H} 12 \mathrm{~A}$ & 0.3623 & 0.7200 & 0.7703 & $0.028^{*}$ \\
\hline H12B & 0.4198 & 0.7622 & 0.8420 & $0.028^{*}$ \\
\hline C13 & 0.4379 (4) & $0.64653(19)$ & $0.84949(18)$ & $0.0249(8)$ \\
\hline H13A & 0.4937 & 0.6513 & 0.8956 & $0.030^{*}$ \\
\hline H13B & 0.3453 & 0.6328 & 0.8592 & $0.030^{*}$ \\
\hline C14 & 0.4705 (4) & $0.51260(18)$ & $0.82625(18)$ & $0.0226(8)$ \\
\hline $\mathrm{C} 15$ & 0.5655 (4) & 0.47385 (19) & $0.87310(18)$ & $0.0251(8)$ \\
\hline C16 & $0.5425(4)$ & $0.3981(2)$ & $0.8873(2)$ & $0.0331(9)$ \\
\hline H16 & 0.6060 & 0.3714 & 0.9186 & $0.040^{*}$ \\
\hline $\mathrm{C} 17$ & $0.4290(5)$ & $0.3613(2)$ & 0.8567 (2) & $0.0381(11)$ \\
\hline
\end{tabular}




\begin{tabular}{|c|c|c|c|c|}
\hline H17 & 0.4165 & 0.3094 & 0.8658 & $0.046^{*}$ \\
\hline C18 & $0.3346(4)$ & $0.3998(2)$ & $0.8133(2)$ & $0.0330(9)$ \\
\hline H18 & 0.2560 & 0.3745 & 0.7933 & $0.040 *$ \\
\hline C19 & $0.3522(4)$ & $0.4757(2)$ & $0.79801(18)$ & $0.0250(8)$ \\
\hline $\mathrm{C} 20$ & $0.6928(4)$ & $0.5109(2)$ & 0.90675 (19) & $0.0271(8)$ \\
\hline $\mathrm{H} 20$ & 0.6865 & 0.5657 & 0.8952 & $0.033^{*}$ \\
\hline $\mathrm{C} 21$ & $0.7086(5)$ & $0.5034(3)$ & $0.9890(2)$ & $0.0440(11)$ \\
\hline $\mathrm{H} 21 \mathrm{~A}$ & 0.6293 & 0.5247 & 1.0085 & $0.066^{*}$ \\
\hline $\mathrm{H} 21 \mathrm{~B}$ & 0.7893 & 0.5305 & 1.0084 & $0.066^{*}$ \\
\hline $\mathrm{H} 21 \mathrm{C}$ & 0.7172 & 0.4502 & 1.0020 & $0.066^{*}$ \\
\hline $\mathrm{C} 22$ & $0.8186(4)$ & $0.4806(2)$ & $0.8764(2)$ & $0.0392(10)$ \\
\hline $\mathrm{H} 22 \mathrm{~A}$ & 0.8278 & 0.4268 & 0.8871 & $0.059 *$ \\
\hline $\mathrm{H} 22 \mathrm{~B}$ & 0.8980 & 0.5074 & 0.8981 & $0.059 *$ \\
\hline $\mathrm{H} 22 \mathrm{C}$ & 0.8106 & 0.4880 & 0.8247 & $0.059 *$ \\
\hline $\mathrm{C} 23$ & $0.2422(4)$ & $0.5165(2)$ & 0.75159 (19) & $0.0283(9)$ \\
\hline $\mathrm{H} 23$ & 0.2722 & 0.5696 & 0.7462 & $0.034^{*}$ \\
\hline $\mathrm{C} 24$ & $0.2197(4)$ & $0.4818(2)$ & $0.6765(2)$ & $0.0389(10)$ \\
\hline $\mathrm{H} 24 \mathrm{~A}$ & 0.3044 & 0.4828 & 0.6541 & $0.058^{*}$ \\
\hline H24B & 0.1512 & 0.5108 & 0.6475 & $0.058^{*}$ \\
\hline $\mathrm{H} 24 \mathrm{C}$ & 0.1893 & 0.4297 & 0.6802 & $0.058 *$ \\
\hline $\mathrm{C} 25$ & 0.1108 (4) & $0.5182(2)$ & $0.7863(2)$ & 0.0414 (11) \\
\hline $\mathrm{H} 25 \mathrm{~A}$ & 0.0446 & 0.5487 & 0.7573 & $0.062 *$ \\
\hline $\mathrm{H} 25 \mathrm{~B}$ & 0.1266 & 0.5402 & 0.8341 & $0.062 *$ \\
\hline $\mathrm{H} 25 \mathrm{C}$ & 0.0764 & 0.4669 & 0.7900 & $0.062 *$ \\
\hline $\mathrm{C} 26$ & $0.9184(4)$ & $0.6953(3)$ & $0.9438(2)$ & $0.0452(11)$ \\
\hline $\mathrm{H} 26 \mathrm{~A}$ & 0.9476 & 0.6495 & 0.9708 & $0.054^{*}$ \\
\hline $\mathrm{H} 26 \mathrm{~B}$ & 0.9545 & 0.7397 & 0.9709 & $0.054^{*}$ \\
\hline $\mathrm{C} 27$ & $0.9679(5)$ & $0.6939(2)$ & $0.8719(2)$ & $0.0455(11)$ \\
\hline $\mathrm{H} 27 \mathrm{~A}$ & 1.0675 & 0.6962 & 0.8768 & $0.055^{*}$ \\
\hline $\mathrm{H} 27 \mathrm{~B}$ & 0.9405 & 0.6461 & 0.8477 & $0.055^{*}$ \\
\hline C28 & $0.9884(4)$ & $0.8245(2)$ & $0.8415(2)$ & $0.0401(10)$ \\
\hline $\mathrm{H} 28 \mathrm{~A}$ & 1.0747 & 0.8222 & 0.8197 & $0.048^{*}$ \\
\hline $\mathrm{H} 28 \mathrm{~B}$ & 1.0079 & 0.8338 & 0.8933 & $0.048^{*}$ \\
\hline C29 & $0.9000(4)$ & $0.8856(2)$ & $0.8075(2)$ & $0.0345(10)$ \\
\hline $\mathrm{H} 29 \mathrm{~A}$ & 0.9423 & 0.9351 & 0.8183 & $0.041 *$ \\
\hline H29B & 0.8903 & 0.8789 & 0.7551 & $0.041 *$ \\
\hline $\mathrm{C} 30$ & $0.7597(4)$ & $0.9279(2)$ & $0.8967(2)$ & $0.0361(10)$ \\
\hline $\mathrm{H} 30 \mathrm{~A}$ & 0.7581 & 0.9821 & 0.8847 & $0.043 *$ \\
\hline H30B & 0.8373 & 0.9181 & 0.9323 & $0.043 *$ \\
\hline C31 & $0.6316(4)$ & $0.9053(2)$ & $0.9252(2)$ & $0.0392(10)$ \\
\hline H31A & 0.6239 & 0.9312 & 0.9712 & $0.047^{*}$ \\
\hline H31B & 0.5545 & 0.9211 & 0.8915 & $0.047^{*}$ \\
\hline C32 & $0.6960(5)$ & $0.8007(2)$ & 1.00139 (19) & $0.0410(11)$ \\
\hline $\mathrm{H} 32 \mathrm{~A}$ & 0.6439 & 0.8133 & 1.0419 & $0.049^{*}$ \\
\hline H32B & 0.7852 & 0.8253 & 1.0091 & $0.049 *$ \\
\hline C33 & $0.7122(5)$ & 0.7179 & $0.9960(2)$ & $0.0458(12)$ \\
\hline H33A & 0.7675 & 0.6991 & 1.0387 & $0.055^{*}$ \\
\hline H33B & 0.6228 & 0.6934 & 0.9941 & $0.055^{*}$ \\
\hline
\end{tabular}


$\begin{array}{lllll}\text { Li1 } & 0.7127(7) & 0.7726(3) & 0.8523(3) & 0.0278(14)\end{array}$

Atomic displacement parameters $\left(\AA^{2}\right)$

\begin{tabular}{|c|c|c|c|c|c|c|}
\hline & $U^{11}$ & $U^{22}$ & $U^{33}$ & $U^{12}$ & $U^{13}$ & $U^{23}$ \\
\hline Sn1 & $0.02406(13)$ & $0.01671(12)$ & $0.01436(11)$ & $0.00045(11)$ & $-0.00054(8)$ & $-0.00026(10)$ \\
\hline $\mathrm{O} 1$ & $0.0362(17)$ & $0.0370(15)$ & $0.0244(14)$ & $-0.0082(13)$ & $-0.0093(12)$ & $0.0053(12)$ \\
\hline $\mathrm{O} 2$ & 0.0389 (17) & $0.0315(15)$ & 0.0302 (14) & $-0.0023(13)$ & 0.0007 (13) & $-0.0025(12)$ \\
\hline $\mathrm{O} 3$ & $0.0337(15)$ & $0.0291(14)$ & $0.0247(13)$ & $-0.0079(12)$ & $0.0034(11)$ & $-0.0044(11)$ \\
\hline $\mathrm{O} 4$ & $0.0347(16)$ & $0.0370(16)$ & $0.0213(13)$ & $-0.0083(13)$ & $0.0008(12)$ & $-0.0036(12)$ \\
\hline N1 & $0.0259(17)$ & 0.0167 (14) & $0.0134(14)$ & $-0.0006(12)$ & $-0.0010(12)$ & $-0.0006(11)$ \\
\hline N2 & $0.0271(17)$ & $0.0167(15)$ & $0.0190(14)$ & $-0.0017(12)$ & $0.0050(13)$ & $-0.0030(12)$ \\
\hline $\mathrm{C} 1$ & $0.025(2)$ & 0.0235 (18) & $0.0161(17)$ & $0.0013(16)$ & $-0.0060(15)$ & $-0.0011(15)$ \\
\hline $\mathrm{C} 2$ & $0.0216(18)$ & 0.0272 (19) & $0.0143(15)$ & $-0.0012(16)$ & $-0.0047(13)$ & $0.0016(15)$ \\
\hline $\mathrm{C} 3$ & $0.030(2)$ & $0.0181(18)$ & $0.0182(17)$ & $-0.0015(15)$ & $-0.0031(16)$ & $-0.0018(14)$ \\
\hline $\mathrm{C} 4$ & 0.0253 (19) & $0.0199(18)$ & $0.0145(15)$ & $0.0028(16)$ & $-0.0033(14)$ & $0.0011(14)$ \\
\hline $\mathrm{C} 5$ & $0.029(2)$ & $0.0232(18)$ & $0.0164(17)$ & $0.0020(16)$ & $-0.0016(15)$ & $-0.0038(14)$ \\
\hline C6 & $0.030(2)$ & $0.038(2)$ & 0.0213 (19) & 0.0017 (17) & $0.0049(16)$ & $-0.0022(16)$ \\
\hline $\mathrm{C} 7$ & $0.030(2)$ & $0.032(2)$ & $0.029(2)$ & $-0.0108(17)$ & $0.0036(18)$ & $0.0006(17)$ \\
\hline $\mathrm{C} 8$ & $0.034(2)$ & 0.0197 (19) & 0.0250 (19) & $-0.0028(16)$ & $-0.0008(17)$ & $-0.0059(15)$ \\
\hline C9 & $0.026(2)$ & $0.0186(17)$ & $0.0131(16)$ & $0.0013(15)$ & $-0.0067(14)$ & $-0.0005(13)$ \\
\hline $\mathrm{C} 10$ & $0.031(2)$ & $0.0213(18)$ & $0.0168(17)$ & $0.0023(16)$ & $-0.0039(15)$ & $0.0013(14)$ \\
\hline $\mathrm{C} 11$ & $0.033(2)$ & $0.0183(18)$ & $0.0184(17)$ & $0.0011(16)$ & $-0.0029(16)$ & $-0.0046(14)$ \\
\hline $\mathrm{C} 12$ & $0.026(2)$ & $0.0223(19)$ & $0.0206(18)$ & $0.0024(16)$ & $0.0031(16)$ & $-0.0038(15)$ \\
\hline C13 & $0.028(2)$ & $0.027(2)$ & 0.0199 (17) & $-0.0024(16)$ & $-0.0001(15)$ & $-0.0042(15)$ \\
\hline C14 & $0.033(2)$ & $0.0181(18)$ & $0.0190(17)$ & $0.0004(16)$ & $0.0139(16)$ & $-0.0018(14)$ \\
\hline $\mathrm{C} 15$ & $0.033(2)$ & $0.0246(19)$ & $0.0195(18)$ & $0.0022(17)$ & $0.0120(16)$ & $0.0016(15)$ \\
\hline $\mathrm{C} 16$ & $0.040(3)$ & $0.029(2)$ & $0.032(2)$ & 0.0072 (19) & 0.0117 (19) & $0.0048(17)$ \\
\hline $\mathrm{C} 17$ & $0.056(3)$ & $0.023(2)$ & $0.039(2)$ & $-0.007(2)$ & $0.025(2)$ & $0.0043(18)$ \\
\hline $\mathrm{C} 18$ & 0.039 (3) & $0.029(2)$ & $0.032(2)$ & -0.0115 & 0.0089 (19) & $-0.0018(17)$ \\
\hline C19 & $0.029(2)$ & 0.0254 (19) & $0.0222(18)$ & $-0.0050(16)$ & $0.0121(16)$ & $-0.0064(15)$ \\
\hline $\mathrm{C} 20$ & $0.030(2)$ & $0.027(2)$ & 0.0251 (19) & 0.0017 (17) & 0.0044 (16) & $0.0071(16)$ \\
\hline $\mathrm{C} 21$ & $0.043(3)$ & $0.061(3)$ & $0.028(2)$ & $-0.002(2)$ & $0.002(2)$ & $0.007(2)$ \\
\hline $\mathrm{C} 22$ & $0.039(3)$ & $0.037(2)$ & $0.044(3)$ & $0.005(2)$ & $0.012(2)$ & $0.007(2)$ \\
\hline $\mathrm{C} 23$ & $0.029(2)$ & $0.025(2)$ & $0.031(2)$ & $-0.0057(16)$ & 0.0043 (18) & $-0.0043(15)$ \\
\hline $\mathrm{C} 24$ & $0.042(3)$ & $0.044(3)$ & $0.031(2)$ & $-0.005(2)$ & $0.0024(19)$ & -0.0105 (19) \\
\hline $\mathrm{C} 25$ & 0.040 & $0.039(2)$ & $0.047(3)$ & $-0.006(2)$ & $0.012(2)$ & $-0.009(2)$ \\
\hline $\mathrm{C} 26$ & $0.041(3)$ & $0.047(3)$ & $0.044(3)$ & $-0.003(2)$ & $-0.012(2)$ & $0.011(2)$ \\
\hline $\mathrm{C} 27$ & $0.043(3)$ & $0.038(2)$ & $0.053(3)$ & $0.006(2)$ & $-0.008(2)$ & $-0.001(2)$ \\
\hline $\mathrm{C} 28$ & $0.036(3)$ & $0.042(3)$ & $0.043(2)$ & $-0.011(2)$ & $0.007(2)$ & $-0.007(2)$ \\
\hline $\mathrm{C} 29$ & $0.040(2)$ & $0.031(2)$ & $0.034(2)$ & $-0.0124(19)$ & $0.0101(19)$ & $0.0029(18)$ \\
\hline $\mathrm{C} 30$ & 0.045 & $0.029(2)$ & $0.035(2)$ & -0.0048 (19) & $0.010(2)$ & $-0.0086(18)$ \\
\hline C31 & $0.044(3)$ & $0.034(2)$ & $0.040(2)$ & $-0.002(2)$ & $0.008(2)$ & $-0.0114(19)$ \\
\hline $\mathrm{C} 32$ & $0.048(3)$ & 0.059 (3) & 0.0156 (19) & $-0.016(2)$ & $0.0013(18)$ & $-0.0004(19)$ \\
\hline $\mathrm{C} 33$ & 0.057 (3) & $0.061(3)$ & $0.017(2)$ & $-0.014(2)$ & $-0.004(2)$ & $0.006(2)$ \\
\hline Li1 & $0.038(4)$ & $0.028(3)$ & $0.017(3)$ & $-0.005(3)$ & $-0.004(3)$ & $0.000(3)$ \\
\hline
\end{tabular}


Geometric parameters $\left(\AA,{ }^{\circ}\right)$

\begin{tabular}{|c|c|c|c|}
\hline $\mathrm{Sn} 1-\mathrm{N} 1$ & $2.157(3)$ & $\mathrm{C} 15-\mathrm{C} 16$ & $1.398(5)$ \\
\hline $\mathrm{Sn} 1-\mathrm{N} 2$ & $2.089(3)$ & $\mathrm{C} 15-\mathrm{C} 20$ & $1.515(5)$ \\
\hline $\mathrm{O} 1-\mathrm{C} 26$ & $1.422(5)$ & C16-H16 & 0.9500 \\
\hline $\mathrm{O} 1-\mathrm{C} 33$ & $1.430(5)$ & $\mathrm{C} 16-\mathrm{C} 17$ & $1.386(6)$ \\
\hline O1-Li1 & $2.068(6)$ & $\mathrm{C} 17-\mathrm{H} 17$ & 0.9500 \\
\hline $\mathrm{O} 2-\mathrm{C} 27$ & $1.438(5)$ & $\mathrm{C} 17-\mathrm{C} 18$ & $1.371(6)$ \\
\hline $\mathrm{O} 2-\mathrm{C} 28$ & $1.430(5)$ & C18-H18 & 0.9500 \\
\hline $\mathrm{O} 2-\mathrm{Li} 1$ & $2.123(7)$ & $\mathrm{C} 18-\mathrm{C} 19$ & $1.395(5)$ \\
\hline $\mathrm{O} 3-\mathrm{C} 29$ & $1.435(4)$ & $\mathrm{C} 19-\mathrm{C} 23$ & $1.521(5)$ \\
\hline $\mathrm{O} 3-\mathrm{C} 30$ & $1.437(4)$ & $\mathrm{C} 20-\mathrm{H} 20$ & 1.0000 \\
\hline O3-Li1 & $2.090(6)$ & $\mathrm{C} 20-\mathrm{C} 21$ & $1.548(5)$ \\
\hline $\mathrm{O} 4-\mathrm{C} 31$ & $1.431(5)$ & $\mathrm{C} 20-\mathrm{C} 22$ & $1.526(5)$ \\
\hline $\mathrm{O} 4-\mathrm{C} 32$ & $1.432(4)$ & $\mathrm{C} 21-\mathrm{H} 21 \mathrm{~A}$ & 0.9800 \\
\hline O4-Li1 & $2.091(7)$ & $\mathrm{C} 21-\mathrm{H} 21 \mathrm{~B}$ & 0.9800 \\
\hline $\mathrm{N} 1-\mathrm{C} 11$ & $1.470(4)$ & $\mathrm{C} 21-\mathrm{H} 21 \mathrm{C}$ & 0.9800 \\
\hline $\mathrm{N} 1-\mathrm{C} 12$ & $1.471(4)$ & $\mathrm{C} 22-\mathrm{H} 22 \mathrm{~A}$ & 0.9800 \\
\hline N1—Li1 & $2.043(7)$ & $\mathrm{C} 22-\mathrm{H} 22 \mathrm{~B}$ & 0.9800 \\
\hline $\mathrm{N} 2-\mathrm{C} 13$ & $1.463(4)$ & $\mathrm{C} 22-\mathrm{H} 22 \mathrm{C}$ & 0.9800 \\
\hline $\mathrm{N} 2-\mathrm{C} 14$ & $1.430(4)$ & $\mathrm{C} 23-\mathrm{H} 23$ & 1.0000 \\
\hline $\mathrm{C} 1-\mathrm{C} 2$ & $1.422(5)$ & $\mathrm{C} 23-\mathrm{C} 24$ & $1.540(5)$ \\
\hline $\mathrm{C} 1-\mathrm{C} 9$ & $1.420(5)$ & $\mathrm{C} 23-\mathrm{C} 25$ & $1.521(5)$ \\
\hline $\mathrm{C} 1-\mathrm{C} 10$ & $1.508(5)$ & $\mathrm{C} 24-\mathrm{H} 24 \mathrm{~A}$ & 0.9800 \\
\hline $\mathrm{C} 2-\mathrm{H} 2$ & 0.9500 & $\mathrm{C} 24-\mathrm{H} 24 \mathrm{~B}$ & 0.9800 \\
\hline $\mathrm{C} 2-\mathrm{C} 3$ & $1.408(5)$ & $\mathrm{C} 24-\mathrm{H} 24 \mathrm{C}$ & 0.9800 \\
\hline $\mathrm{C} 3-\mathrm{H} 3$ & 0.9500 & $\mathrm{C} 25-\mathrm{H} 25 \mathrm{~A}$ & 0.9800 \\
\hline $\mathrm{C} 3-\mathrm{C} 4$ & $1.411(5)$ & $\mathrm{C} 25-\mathrm{H} 25 \mathrm{~B}$ & 0.9800 \\
\hline $\mathrm{C} 4-\mathrm{C} 5$ & $1.411(5)$ & $\mathrm{C} 25-\mathrm{H} 25 \mathrm{C}$ & 0.9800 \\
\hline $\mathrm{C} 4-\mathrm{C} 9$ & $1.452(4)$ & $\mathrm{C} 26-\mathrm{H} 26 \mathrm{~A}$ & 0.9900 \\
\hline $\mathrm{C} 5-\mathrm{H} 5$ & 0.9500 & $\mathrm{C} 26-\mathrm{H} 26 \mathrm{~B}$ & 0.9900 \\
\hline $\mathrm{C} 5-\mathrm{C} 6$ & $1.374(5)$ & $\mathrm{C} 26-\mathrm{C} 27$ & $1.486(6)$ \\
\hline C6-H6 & 0.9500 & $\mathrm{C} 27-\mathrm{H} 27 \mathrm{~A}$ & 0.9900 \\
\hline $\mathrm{C} 6-\mathrm{C} 7$ & $1.418(5)$ & $\mathrm{C} 27-\mathrm{H} 27 \mathrm{~B}$ & 0.9900 \\
\hline $\mathrm{C} 7-\mathrm{H} 7$ & 0.9500 & $\mathrm{C} 28-\mathrm{H} 28 \mathrm{~A}$ & 0.9900 \\
\hline $\mathrm{C} 7-\mathrm{C} 8$ & $1.367(5)$ & $\mathrm{C} 28-\mathrm{H} 28 \mathrm{~B}$ & 0.9900 \\
\hline $\mathrm{C} 8-\mathrm{H} 8$ & 0.9500 & $\mathrm{C} 28-\mathrm{C} 29$ & $1.504(6)$ \\
\hline $\mathrm{C} 8-\mathrm{C} 9$ & $1.400(5)$ & $\mathrm{C} 29-\mathrm{H} 29 \mathrm{~A}$ & 0.9900 \\
\hline $\mathrm{C} 10-\mathrm{H} 10 \mathrm{~A}$ & 0.9900 & $\mathrm{C} 29-\mathrm{H} 29 \mathrm{~B}$ & 0.9900 \\
\hline $\mathrm{C} 10-\mathrm{H} 10 \mathrm{~B}$ & 0.9900 & $\mathrm{C} 30-\mathrm{H} 30 \mathrm{~A}$ & 0.9900 \\
\hline $\mathrm{C} 10-\mathrm{C} 11$ & $1.542(5)$ & $\mathrm{C} 30-\mathrm{H} 30 \mathrm{~B}$ & 0.9900 \\
\hline C11-H11A & 0.9900 & $\mathrm{C} 30-\mathrm{C} 31$ & $1.489(5)$ \\
\hline C11-H11B & 0.9900 & $\mathrm{C} 31-\mathrm{H} 31 \mathrm{~A}$ & 0.9900 \\
\hline $\mathrm{C} 12-\mathrm{H} 12 \mathrm{~A}$ & 0.9900 & $\mathrm{C} 31-\mathrm{H} 31 \mathrm{~B}$ & 0.9900 \\
\hline $\mathrm{C} 12-\mathrm{H} 12 \mathrm{~B}$ & 0.9900 & $\mathrm{C} 32-\mathrm{H} 32 \mathrm{~A}$ & 0.9900 \\
\hline $\mathrm{C} 12-\mathrm{C} 13$ & $1.517(5)$ & $\mathrm{C} 32-\mathrm{H} 32 \mathrm{~B}$ & 0.9900 \\
\hline $\mathrm{C} 13-\mathrm{H} 13 \mathrm{~A}$ & 0.9900 & $\mathrm{C} 32-\mathrm{C} 33$ & $1.487(6)$ \\
\hline C13-H13B & 0.9900 & C $33-\mathrm{H} 33 \mathrm{~A}$ & 0.9900 \\
\hline
\end{tabular}




\begin{tabular}{|c|c|c|c|}
\hline $\mathrm{C} 14-\mathrm{C} 15$ & $1.412(5)$ & C $33-\mathrm{H} 33 \mathrm{~B}$ & 0.9900 \\
\hline $\mathrm{C} 14-\mathrm{C} 19$ & $1.410(5)$ & & \\
\hline $\mathrm{N} 2-\mathrm{Sn} 1-\mathrm{N} 1$ & $79.49(10)$ & $\mathrm{C} 15-\mathrm{C} 20-\mathrm{C} 22$ & $112.1(3)$ \\
\hline $\mathrm{C} 26-\mathrm{O} 1-\mathrm{C} 33$ & $114.4(3)$ & $\mathrm{C} 21-\mathrm{C} 20-\mathrm{H} 20$ & 107.5 \\
\hline $\mathrm{C} 26-\mathrm{O} 1-\mathrm{Li} 1$ & $110.9(3)$ & $\mathrm{C} 22-\mathrm{C} 20-\mathrm{H} 20$ & 107.5 \\
\hline $\mathrm{C} 33-\mathrm{O} 1-\mathrm{Li} 1$ & $109.3(3)$ & $\mathrm{C} 22-\mathrm{C} 20-\mathrm{C} 21$ & $109.6(3)$ \\
\hline $\mathrm{C} 27-\mathrm{O} 2-\mathrm{Li} 1$ & $107.4(3)$ & $\mathrm{C} 20-\mathrm{C} 21-\mathrm{H} 21 \mathrm{~A}$ & 109.5 \\
\hline $\mathrm{C} 28-\mathrm{O} 2-\mathrm{C} 27$ & $114.2(3)$ & $\mathrm{C} 20-\mathrm{C} 21-\mathrm{H} 21 \mathrm{~B}$ & 109.5 \\
\hline $\mathrm{C} 28-\mathrm{O} 2-\mathrm{Li} 1$ & $109.4(3)$ & $\mathrm{C} 20-\mathrm{C} 21-\mathrm{H} 21 \mathrm{C}$ & 109.5 \\
\hline $\mathrm{C} 29-\mathrm{O} 3-\mathrm{C} 30$ & $114.0(3)$ & $\mathrm{H} 21 \mathrm{~A}-\mathrm{C} 21-\mathrm{H} 21 \mathrm{~B}$ & 109.5 \\
\hline $\mathrm{C} 29-\mathrm{O} 3-\mathrm{Li} 1$ & $110.8(3)$ & $\mathrm{H} 21 \mathrm{~A}-\mathrm{C} 21-\mathrm{H} 21 \mathrm{C}$ & 109.5 \\
\hline $\mathrm{C} 30-\mathrm{O} 3-\mathrm{Li} 1$ & $109.9(3)$ & $\mathrm{H} 21 \mathrm{~B}-\mathrm{C} 21-\mathrm{H} 21 \mathrm{C}$ & 109.5 \\
\hline $\mathrm{C} 31-\mathrm{O} 4-\mathrm{C} 32$ & $113.9(3)$ & $\mathrm{C} 20-\mathrm{C} 22-\mathrm{H} 22 \mathrm{~A}$ & 109.5 \\
\hline $\mathrm{C} 31-\mathrm{O} 4-\mathrm{Li} 1$ & $108.6(3)$ & $\mathrm{C} 20-\mathrm{C} 22-\mathrm{H} 22 \mathrm{~B}$ & 109.5 \\
\hline $\mathrm{C} 32-\mathrm{O} 4-\mathrm{Li} 1$ & $107.8(3)$ & $\mathrm{C} 20-\mathrm{C} 22-\mathrm{H} 22 \mathrm{C}$ & 109.5 \\
\hline $\mathrm{C} 11-\mathrm{N} 1-\mathrm{Sn} 1$ & $112.15(19)$ & $\mathrm{H} 22 \mathrm{~A}-\mathrm{C} 22-\mathrm{H} 22 \mathrm{~B}$ & 109.5 \\
\hline $\mathrm{C} 11-\mathrm{N} 1-\mathrm{C} 12$ & $111.9(3)$ & $\mathrm{H} 22 \mathrm{~A}-\mathrm{C} 22-\mathrm{H} 22 \mathrm{C}$ & 109.5 \\
\hline $\mathrm{C} 11-\mathrm{N} 1-\mathrm{Li} 1$ & $100.8(3)$ & $\mathrm{H} 22 \mathrm{~B}-\mathrm{C} 22-\mathrm{H} 22 \mathrm{C}$ & 109.5 \\
\hline $\mathrm{C} 12-\mathrm{N} 1-\mathrm{Sn} 1$ & $108.64(19)$ & $\mathrm{C} 19-\mathrm{C} 23-\mathrm{H} 23$ & 107.7 \\
\hline $\mathrm{C} 12-\mathrm{N} 1-\mathrm{Li} 1$ & $113.1(3)$ & $\mathrm{C} 19-\mathrm{C} 23-\mathrm{C} 24$ & $111.7(3)$ \\
\hline Li1-N1-Sn1 & $110.2(2)$ & $\mathrm{C} 19-\mathrm{C} 23-\mathrm{C} 25$ & $111.5(3)$ \\
\hline $\mathrm{C} 13-\mathrm{N} 2-\mathrm{Sn} 1$ & $115.1(2)$ & $\mathrm{C} 24-\mathrm{C} 23-\mathrm{H} 23$ & 107.7 \\
\hline $\mathrm{C} 14-\mathrm{N} 2-\mathrm{Sn} 1$ & $127.8(2)$ & $\mathrm{C} 25-\mathrm{C} 23-\mathrm{H} 23$ & 107.7 \\
\hline $\mathrm{C} 14-\mathrm{N} 2-\mathrm{C} 13$ & $115.8(3)$ & $\mathrm{C} 25-\mathrm{C} 23-\mathrm{C} 24$ & $110.4(3)$ \\
\hline $\mathrm{C} 2-\mathrm{C} 1-\mathrm{C} 10$ & $127.4(3)$ & $\mathrm{C} 23-\mathrm{C} 24-\mathrm{H} 24 \mathrm{~A}$ & 109.5 \\
\hline $\mathrm{C} 9-\mathrm{C} 1-\mathrm{C} 2$ & $106.8(3)$ & $\mathrm{C} 23-\mathrm{C} 24-\mathrm{H} 24 \mathrm{~B}$ & 109.5 \\
\hline $\mathrm{C} 9-\mathrm{C} 1-\mathrm{C} 10$ & $125.7(3)$ & $\mathrm{C} 23-\mathrm{C} 24-\mathrm{H} 24 \mathrm{C}$ & 109.5 \\
\hline $\mathrm{C} 1-\mathrm{C} 2-\mathrm{H} 2$ & 125.1 & $\mathrm{H} 24 \mathrm{~A}-\mathrm{C} 24-\mathrm{H} 24 \mathrm{~B}$ & 109.5 \\
\hline $\mathrm{C} 3-\mathrm{C} 2-\mathrm{C} 1$ & $109.8(3)$ & $\mathrm{H} 24 \mathrm{~A}-\mathrm{C} 24-\mathrm{H} 24 \mathrm{C}$ & 109.5 \\
\hline $\mathrm{C} 3-\mathrm{C} 2-\mathrm{H} 2$ & 125.1 & $\mathrm{H} 24 \mathrm{~B}-\mathrm{C} 24-\mathrm{H} 24 \mathrm{C}$ & 109.5 \\
\hline $\mathrm{C} 2-\mathrm{C} 3-\mathrm{H} 3$ & 126.0 & $\mathrm{C} 23-\mathrm{C} 25-\mathrm{H} 25 \mathrm{~A}$ & 109.5 \\
\hline $\mathrm{C} 2-\mathrm{C} 3-\mathrm{C} 4$ & $107.9(3)$ & $\mathrm{C} 23-\mathrm{C} 25-\mathrm{H} 25 \mathrm{~B}$ & 109.5 \\
\hline $\mathrm{C} 4-\mathrm{C} 3-\mathrm{H} 3$ & 126.0 & $\mathrm{C} 23-\mathrm{C} 25-\mathrm{H} 25 \mathrm{C}$ & 109.5 \\
\hline $\mathrm{C} 3-\mathrm{C} 4-\mathrm{C} 9$ & $107.5(3)$ & $\mathrm{H} 25 \mathrm{~A}-\mathrm{C} 25-\mathrm{H} 25 \mathrm{~B}$ & 109.5 \\
\hline $\mathrm{C} 5-\mathrm{C} 4-\mathrm{C} 3$ & $133.8(3)$ & $\mathrm{H} 25 \mathrm{~A}-\mathrm{C} 25-\mathrm{H} 25 \mathrm{C}$ & 109.5 \\
\hline $\mathrm{C} 5-\mathrm{C} 4-\mathrm{C} 9$ & $118.7(3)$ & $\mathrm{H} 25 \mathrm{~B}-\mathrm{C} 25-\mathrm{H} 25 \mathrm{C}$ & 109.5 \\
\hline $\mathrm{C} 4-\mathrm{C} 5-\mathrm{H} 5$ & 119.9 & $\mathrm{O} 1-\mathrm{C} 26-\mathrm{H} 26 \mathrm{~A}$ & 110.3 \\
\hline $\mathrm{C} 6-\mathrm{C} 5-\mathrm{C} 4$ & $120.1(3)$ & $\mathrm{O} 1-\mathrm{C} 26-\mathrm{H} 26 \mathrm{~B}$ & 110.3 \\
\hline $\mathrm{C} 6-\mathrm{C} 5-\mathrm{H} 5$ & 119.9 & $\mathrm{O} 1-\mathrm{C} 26-\mathrm{C} 27$ & $107.0(3)$ \\
\hline $\mathrm{C} 5-\mathrm{C} 6-\mathrm{H} 6$ & 119.6 & $\mathrm{H} 26 \mathrm{~A}-\mathrm{C} 26-\mathrm{H} 26 \mathrm{~B}$ & 108.6 \\
\hline $\mathrm{C} 5-\mathrm{C} 6-\mathrm{C} 7$ & $120.8(3)$ & $\mathrm{C} 27-\mathrm{C} 26-\mathrm{H} 26 \mathrm{~A}$ & 110.3 \\
\hline $\mathrm{C} 7-\mathrm{C} 6-\mathrm{H} 6$ & 119.6 & $\mathrm{C} 27-\mathrm{C} 26-\mathrm{H} 26 \mathrm{~B}$ & 110.3 \\
\hline $\mathrm{C} 6-\mathrm{C} 7-\mathrm{H} 7$ & 119.8 & $\mathrm{O} 2-\mathrm{C} 27-\mathrm{C} 26$ & $111.0(4)$ \\
\hline $\mathrm{C} 8-\mathrm{C} 7-\mathrm{C} 6$ & $120.5(3)$ & $\mathrm{O} 2-\mathrm{C} 27-\mathrm{H} 27 \mathrm{~A}$ & 109.4 \\
\hline $\mathrm{C} 8-\mathrm{C} 7-\mathrm{H} 7$ & 119.8 & $\mathrm{O} 2-\mathrm{C} 27-\mathrm{H} 27 \mathrm{~B}$ & 109.4 \\
\hline $\mathrm{C} 7-\mathrm{C} 8-\mathrm{H} 8$ & 119.7 & $\mathrm{C} 26-\mathrm{C} 27-\mathrm{H} 27 \mathrm{~A}$ & 109.4 \\
\hline $\mathrm{C} 7-\mathrm{C} 8-\mathrm{C} 9$ & $120.5(3)$ & $\mathrm{C} 26-\mathrm{C} 27-\mathrm{H} 27 \mathrm{~B}$ & 109.4 \\
\hline
\end{tabular}




\begin{tabular}{|c|c|}
\hline $\mathrm{C} 9-\mathrm{C} 8-\mathrm{H} 8$ & 119.7 \\
\hline $\mathrm{C} 1-\mathrm{C} 9-\mathrm{C} 4$ & $108.0(3)$ \\
\hline $\mathrm{C} 8-\mathrm{C} 9-\mathrm{C} 1$ & $132.5(3)$ \\
\hline $\mathrm{C} 8-\mathrm{C} 9-\mathrm{C} 4$ & $119.4(3)$ \\
\hline $\mathrm{C} 1-\mathrm{C} 10-\mathrm{H} 10 \mathrm{~A}$ & 108.9 \\
\hline $\mathrm{C} 1-\mathrm{C} 10-\mathrm{H} 10 \mathrm{~B}$ & 108.9 \\
\hline $\mathrm{C} 1-\mathrm{C} 10-\mathrm{C} 11$ & $113.3(3)$ \\
\hline $\mathrm{H} 10 \mathrm{~A}-\mathrm{C} 10-\mathrm{H} 10 \mathrm{~B}$ & 107.7 \\
\hline $\mathrm{C} 11-\mathrm{C} 10-\mathrm{H} 10 \mathrm{~A}$ & 108.9 \\
\hline $\mathrm{C} 11-\mathrm{C} 10-\mathrm{H} 10 \mathrm{~B}$ & 108.9 \\
\hline $\mathrm{N} 1-\mathrm{C} 11-\mathrm{C} 10$ & $114.7(3)$ \\
\hline $\mathrm{N} 1-\mathrm{C} 11-\mathrm{H} 11 \mathrm{~A}$ & 108.6 \\
\hline $\mathrm{N} 1-\mathrm{C} 11-\mathrm{H} 11 \mathrm{~B}$ & 108.6 \\
\hline $\mathrm{C} 10-\mathrm{C} 11-\mathrm{H} 11 \mathrm{~A}$ & 108.6 \\
\hline $\mathrm{C} 10-\mathrm{C} 11-\mathrm{H} 11 \mathrm{~B}$ & 108.6 \\
\hline $\mathrm{H} 11 \mathrm{~A}-\mathrm{C} 11-\mathrm{H} 11 \mathrm{~B}$ & 107.6 \\
\hline $\mathrm{N} 1-\mathrm{C} 12-\mathrm{H} 12 \mathrm{~A}$ & 109.6 \\
\hline $\mathrm{N} 1-\mathrm{C} 12-\mathrm{H} 12 \mathrm{~B}$ & 109.6 \\
\hline $\mathrm{N} 1-\mathrm{C} 12-\mathrm{C} 13$ & $110.3(3)$ \\
\hline $\mathrm{H} 12 \mathrm{~A}-\mathrm{C} 12-\mathrm{H} 12 \mathrm{~B}$ & 108.1 \\
\hline $\mathrm{C} 13-\mathrm{C} 12-\mathrm{H} 12 \mathrm{~A}$ & 109.6 \\
\hline $\mathrm{C} 13-\mathrm{C} 12-\mathrm{H} 12 \mathrm{~B}$ & 109.6 \\
\hline $\mathrm{N} 2-\mathrm{C} 13-\mathrm{C} 12$ & $108.5(3)$ \\
\hline $\mathrm{N} 2-\mathrm{C} 13-\mathrm{H} 13 \mathrm{~A}$ & 110.0 \\
\hline $\mathrm{N} 2-\mathrm{C} 13-\mathrm{H} 13 \mathrm{~B}$ & 110.0 \\
\hline $\mathrm{C} 12-\mathrm{C} 13-\mathrm{H} 13 \mathrm{~A}$ & 110.0 \\
\hline $\mathrm{C} 12-\mathrm{C} 13-\mathrm{H} 13 \mathrm{~B}$ & 110.0 \\
\hline $\mathrm{H} 13 \mathrm{~A}-\mathrm{C} 13-\mathrm{H} 13 \mathrm{~B}$ & 108.4 \\
\hline $\mathrm{C} 15-\mathrm{C} 14-\mathrm{N} 2$ & $121.0(3)$ \\
\hline $\mathrm{C} 19-\mathrm{C} 14-\mathrm{N} 2$ & $119.3(3)$ \\
\hline $\mathrm{C} 19-\mathrm{C} 14-\mathrm{C} 15$ & $119.7(3)$ \\
\hline $\mathrm{C} 14-\mathrm{C} 15-\mathrm{C} 20$ & $122.3(3)$ \\
\hline $\mathrm{C} 16-\mathrm{C} 15-\mathrm{C} 14$ & $118.6(4)$ \\
\hline $\mathrm{C} 16-\mathrm{C} 15-\mathrm{C} 20$ & $119.1(3)$ \\
\hline $\mathrm{C} 15-\mathrm{C} 16-\mathrm{H} 16$ & 119.4 \\
\hline $\mathrm{C} 17-\mathrm{C} 16-\mathrm{C} 15$ & $121.3(4)$ \\
\hline $\mathrm{C} 17-\mathrm{C} 16-\mathrm{H} 16$ & 119.4 \\
\hline $\mathrm{C} 16-\mathrm{C} 17-\mathrm{H} 17$ & 120.1 \\
\hline $\mathrm{C} 18-\mathrm{C} 17-\mathrm{C} 16$ & $119.9(4)$ \\
\hline $\mathrm{C} 18-\mathrm{C} 17-\mathrm{H} 17$ & 120.1 \\
\hline $\mathrm{C} 17-\mathrm{C} 18-\mathrm{H} 18$ & 119.5 \\
\hline $\mathrm{C} 17-\mathrm{C} 18-\mathrm{C} 19$ & $121.0(4)$ \\
\hline $\mathrm{C} 19-\mathrm{C} 18-\mathrm{H} 18$ & 119.5 \\
\hline $\mathrm{C} 14-\mathrm{C} 19-\mathrm{C} 23$ & $121.7(3)$ \\
\hline $\mathrm{C} 18-\mathrm{C} 19-\mathrm{C} 14$ & $119.4(4)$ \\
\hline $\mathrm{C} 18-\mathrm{C} 19-\mathrm{C} 23$ & $118.9(3)$ \\
\hline $\mathrm{C} 15-\mathrm{C} 20-\mathrm{H} 20$ & 107.5 \\
\hline $\mathrm{C} 15-\mathrm{C} 20-\mathrm{C} 21$ & $112.4(3)$ \\
\hline
\end{tabular}

\begin{tabular}{|c|c|}
\hline $\mathrm{H} 27 \mathrm{~A}-\mathrm{C} 27-\mathrm{H} 27 \mathrm{~B}$ & 108.0 \\
\hline $\mathrm{O} 2-\mathrm{C} 28-\mathrm{H} 28 \mathrm{~A}$ & 110.5 \\
\hline $\mathrm{O} 2-\mathrm{C} 28-\mathrm{H} 28 \mathrm{~B}$ & 110.5 \\
\hline $\mathrm{O} 2-\mathrm{C} 28-\mathrm{C} 29$ & $106.0(3)$ \\
\hline $\mathrm{H} 28 \mathrm{~A}-\mathrm{C} 28-\mathrm{H} 28 \mathrm{~B}$ & 108.7 \\
\hline $\mathrm{C} 29-\mathrm{C} 28-\mathrm{H} 28 \mathrm{~A}$ & 110.5 \\
\hline $\mathrm{C} 29-\mathrm{C} 28-\mathrm{H} 28 \mathrm{~B}$ & 110.5 \\
\hline $\mathrm{O} 3-\mathrm{C} 29-\mathrm{C} 28$ & $110.2(3)$ \\
\hline $\mathrm{O} 3-\mathrm{C} 29-\mathrm{H} 29 \mathrm{~A}$ & 109.6 \\
\hline $\mathrm{O} 3-\mathrm{C} 29-\mathrm{H} 29 \mathrm{~B}$ & 109.6 \\
\hline $\mathrm{C} 28-\mathrm{C} 29-\mathrm{H} 29 \mathrm{~A}$ & 109.6 \\
\hline $\mathrm{C} 28-\mathrm{C} 29-\mathrm{H} 29 \mathrm{~B}$ & 109.6 \\
\hline $\mathrm{H} 29 \mathrm{~A}-\mathrm{C} 29-\mathrm{H} 29 \mathrm{~B}$ & 108.1 \\
\hline $\mathrm{O} 3-\mathrm{C} 30-\mathrm{H} 30 \mathrm{~A}$ & 110.5 \\
\hline $\mathrm{O} 3-\mathrm{C} 30-\mathrm{H} 30 \mathrm{~B}$ & 110.5 \\
\hline $\mathrm{O} 3-\mathrm{C} 30-\mathrm{C} 31$ & $106.2(3)$ \\
\hline $\mathrm{H} 30 \mathrm{~A}-\mathrm{C} 30-\mathrm{H} 30 \mathrm{~B}$ & 108.7 \\
\hline $\mathrm{C} 31-\mathrm{C} 30-\mathrm{H} 30 \mathrm{~A}$ & 110.5 \\
\hline $\mathrm{C} 31-\mathrm{C} 30-\mathrm{H} 30 \mathrm{~B}$ & 110.5 \\
\hline $\mathrm{O} 4-\mathrm{C} 31-\mathrm{C} 30$ & $111.3(3)$ \\
\hline $\mathrm{O} 4-\mathrm{C} 31-\mathrm{H} 31 \mathrm{~A}$ & 109.4 \\
\hline $\mathrm{O} 4-\mathrm{C} 31-\mathrm{H} 31 \mathrm{~B}$ & 109.4 \\
\hline $\mathrm{C} 30-\mathrm{C} 31-\mathrm{H} 31 \mathrm{~A}$ & 109.4 \\
\hline $\mathrm{C} 30-\mathrm{C} 31-\mathrm{H} 31 \mathrm{~B}$ & 109.4 \\
\hline $\mathrm{H} 31 \mathrm{~A}-\mathrm{C} 31-\mathrm{H} 31 \mathrm{~B}$ & 108.0 \\
\hline $\mathrm{O} 4-\mathrm{C} 32-\mathrm{H} 32 \mathrm{~A}$ & 110.3 \\
\hline $\mathrm{O} 4-\mathrm{C} 32-\mathrm{H} 32 \mathrm{~B}$ & 110.3 \\
\hline $\mathrm{O} 4-\mathrm{C} 32-\mathrm{C} 33$ & $107.3(3)$ \\
\hline $\mathrm{H} 32 \mathrm{~A}-\mathrm{C} 32-\mathrm{H} 32 \mathrm{~B}$ & 108.5 \\
\hline $\mathrm{C} 33-\mathrm{C} 32-\mathrm{H} 32 \mathrm{~A}$ & 110.3 \\
\hline $\mathrm{C} 33-\mathrm{C} 32-\mathrm{H} 32 \mathrm{~B}$ & 110.3 \\
\hline $\mathrm{O} 1-\mathrm{C} 33-\mathrm{C} 32$ & $110.6(3)$ \\
\hline $\mathrm{O} 1-\mathrm{C} 33-\mathrm{H} 33 \mathrm{~A}$ & 109.5 \\
\hline $\mathrm{O} 1-\mathrm{C} 33-\mathrm{H} 33 \mathrm{~B}$ & 109.5 \\
\hline $\mathrm{C} 32-\mathrm{C} 33-\mathrm{H} 33 \mathrm{~A}$ & 109.5 \\
\hline $\mathrm{C} 32-\mathrm{C} 33-\mathrm{H} 33 \mathrm{~B}$ & 109.5 \\
\hline $\mathrm{H} 33 \mathrm{~A}-\mathrm{C} 33-\mathrm{H} 33 \mathrm{~B}$ & 108.1 \\
\hline $\mathrm{O} 1-\mathrm{Li} 1-\mathrm{O} 2$ & $80.6(2)$ \\
\hline $\mathrm{O} 1-\mathrm{Li} 1-\mathrm{O} 3$ & $131.0(3)$ \\
\hline $\mathrm{O} 1-\mathrm{Li} 1-\mathrm{O} 4$ & $81.4(2)$ \\
\hline $\mathrm{O} 3-\mathrm{Li} 1-\mathrm{O} 2$ & $79.5(2)$ \\
\hline $\mathrm{O} 3-\mathrm{Li} 1-\mathrm{O} 4$ & $80.7(2)$ \\
\hline $\mathrm{O} 4-\mathrm{Li} 1-\mathrm{O} 2$ & $133.3(3)$ \\
\hline $\mathrm{N} 1-\mathrm{Li} 1-\mathrm{O} 1$ & $114.9(3)$ \\
\hline $\mathrm{N} 1-\mathrm{Li} 1-\mathrm{O} 2$ & $116.7(3)$ \\
\hline $\mathrm{N} 1-\mathrm{Li} 1-\mathrm{O} 3$ & $114.1(3)$ \\
\hline $\mathrm{N} 1-\mathrm{Li} 1-\mathrm{O} 4$ & $110.0(3)$ \\
\hline
\end{tabular}




\begin{tabular}{|c|c|c|c|}
\hline $\mathrm{Sn} 1-\mathrm{N} 1-\mathrm{C} 11-\mathrm{C} 10$ & $54.6(3)$ & $\mathrm{C} 13-\mathrm{N} 2-\mathrm{C} 14-\mathrm{C} 15$ & $-92.8(4)$ \\
\hline $\mathrm{Sn} 1-\mathrm{N} 1-\mathrm{C} 12-\mathrm{C} 13$ & $41.9(3)$ & $\mathrm{C} 13-\mathrm{N} 2-\mathrm{C} 14-\mathrm{C} 19$ & $88.4(4)$ \\
\hline $\mathrm{Sn} 1-\mathrm{N} 2-\mathrm{C} 13-\mathrm{C} 12$ & $27.7(3)$ & $\mathrm{C} 14-\mathrm{N} 2-\mathrm{C} 13-\mathrm{C} 12$ & $-164.2(3)$ \\
\hline $\mathrm{Sn} 1-\mathrm{N} 2-\mathrm{C} 14-\mathrm{C} 15$ & $73.5(4)$ & $\mathrm{C} 14-\mathrm{C} 15-\mathrm{C} 16-\mathrm{C} 17$ & $-0.4(5)$ \\
\hline $\mathrm{Sn} 1-\mathrm{N} 2-\mathrm{C} 14-\mathrm{C} 19$ & $-105.3(3)$ & $\mathrm{C} 14-\mathrm{C} 15-\mathrm{C} 20-\mathrm{C} 21$ & $126.3(4)$ \\
\hline $\mathrm{O} 1-\mathrm{C} 26-\mathrm{C} 27-\mathrm{O} 2$ & $54.0(5)$ & $\mathrm{C} 14-\mathrm{C} 15-\mathrm{C} 20-\mathrm{C} 22$ & $-109.8(4)$ \\
\hline $\mathrm{O} 2-\mathrm{C} 28-\mathrm{C} 29-\mathrm{O} 3$ & $53.8(4)$ & $\mathrm{C} 14-\mathrm{C} 19-\mathrm{C} 23-\mathrm{C} 24$ & $118.9(4)$ \\
\hline $\mathrm{O} 3-\mathrm{C} 30-\mathrm{C} 31-\mathrm{O} 4$ & $54.0(4)$ & $\mathrm{C} 14-\mathrm{C} 19-\mathrm{C} 23-\mathrm{C} 25$ & $-117.1(4)$ \\
\hline $\mathrm{O} 4-\mathrm{C} 32-\mathrm{C} 33-\mathrm{O} 1$ & $54.1(5)$ & $\mathrm{C} 15-\mathrm{C} 14-\mathrm{C} 19-\mathrm{C} 18$ & $-4.0(5)$ \\
\hline $\mathrm{N} 1-\mathrm{C} 12-\mathrm{C} 13-\mathrm{N} 2$ & $-45.8(4)$ & $\mathrm{C} 15-\mathrm{C} 14-\mathrm{C} 19-\mathrm{C} 23$ & $175.6(3)$ \\
\hline $\mathrm{N} 2-\mathrm{C} 14-\mathrm{C} 15-\mathrm{C} 16$ & $-175.5(3)$ & $\mathrm{C} 15-\mathrm{C} 16-\mathrm{C} 17-\mathrm{C} 18$ & $-2.0(6)$ \\
\hline $\mathrm{N} 2-\mathrm{C} 14-\mathrm{C} 15-\mathrm{C} 20$ & $2.8(5)$ & $\mathrm{C} 16-\mathrm{C} 15-\mathrm{C} 20-\mathrm{C} 21$ & $-55.4(4)$ \\
\hline $\mathrm{N} 2-\mathrm{C} 14-\mathrm{C} 19-\mathrm{C} 18$ & $174.8(3)$ & $\mathrm{C} 16-\mathrm{C} 15-\mathrm{C} 20-\mathrm{C} 22$ & $68.5(4)$ \\
\hline $\mathrm{N} 2-\mathrm{C} 14-\mathrm{C} 19-\mathrm{C} 23$ & $-5.6(5)$ & $\mathrm{C} 16-\mathrm{C} 17-\mathrm{C} 18-\mathrm{C} 19$ & $1.3(6)$ \\
\hline $\mathrm{C} 1-\mathrm{C} 2-\mathrm{C} 3-\mathrm{C} 4$ & $0.0(4)$ & $\mathrm{C} 17-\mathrm{C} 18-\mathrm{C} 19-\mathrm{C} 14$ & $1.7(5)$ \\
\hline $\mathrm{C} 1-\mathrm{C} 10-\mathrm{C} 11-\mathrm{N} 1$ & $-54.3(4)$ & $\mathrm{C} 17-\mathrm{C} 18-\mathrm{C} 19-\mathrm{C} 23$ & $-177.9(3)$ \\
\hline $\mathrm{C} 2-\mathrm{C} 1-\mathrm{C} 9-\mathrm{C} 4$ & $0.6(4)$ & $\mathrm{C} 18-\mathrm{C} 19-\mathrm{C} 23-\mathrm{C} 24$ & $-61.6(4)$ \\
\hline $\mathrm{C} 2-\mathrm{C} 1-\mathrm{C} 9-\mathrm{C} 8$ & $177.3(4)$ & $\mathrm{C} 18-\mathrm{C} 19-\mathrm{C} 23-\mathrm{C} 25$ & $62.4(4)$ \\
\hline $\mathrm{C} 2-\mathrm{C} 1-\mathrm{C} 10-\mathrm{C} 11$ & $102.2(4)$ & $\mathrm{C} 19-\mathrm{C} 14-\mathrm{C} 15-\mathrm{C} 16$ & $3.3(5)$ \\
\hline $\mathrm{C} 2-\mathrm{C} 3-\mathrm{C} 4-\mathrm{C} 5$ & $-179.4(3)$ & $\mathrm{C} 19-\mathrm{C} 14-\mathrm{C} 15-\mathrm{C} 20$ & $-178.4(3)$ \\
\hline $\mathrm{C} 2-\mathrm{C} 3-\mathrm{C} 4-\mathrm{C} 9$ & $0.4(4)$ & $\mathrm{C} 20-\mathrm{C} 15-\mathrm{C} 16-\mathrm{C} 17$ & $-178.7(3)$ \\
\hline $\mathrm{C} 3-\mathrm{C} 4-\mathrm{C} 5-\mathrm{C} 6$ & $177.9(4)$ & $\mathrm{C} 26-\mathrm{O} 1-\mathrm{C} 33-\mathrm{C} 32$ & $90.1(4)$ \\
\hline $\mathrm{C} 3-\mathrm{C} 4-\mathrm{C} 9-\mathrm{C} 1$ & $-0.6(4)$ & $\mathrm{C} 27-\mathrm{O} 2-\mathrm{C} 28-\mathrm{C} 29$ & $-166.0(3)$ \\
\hline $\mathrm{C} 3-\mathrm{C} 4-\mathrm{C} 9-\mathrm{C} 8$ & $-177.8(3)$ & $\mathrm{C} 28-\mathrm{O} 2-\mathrm{C} 27-\mathrm{C} 26$ & $83.4(4)$ \\
\hline $\mathrm{C} 4-\mathrm{C} 5-\mathrm{C} 6-\mathrm{C} 7$ & $0.5(5)$ & $\mathrm{C} 29-\mathrm{O} 3-\mathrm{C} 30-\mathrm{C} 31$ & $-167.3(3)$ \\
\hline $\mathrm{C} 5-\mathrm{C} 4-\mathrm{C} 9-\mathrm{C} 1$ & $179.2(3)$ & $\mathrm{C} 30-\mathrm{O} 3-\mathrm{C} 29-\mathrm{C} 28$ & $89.4(4)$ \\
\hline $\mathrm{C} 5-\mathrm{C} 4-\mathrm{C} 9-\mathrm{C} 8$ & $2.0(5)$ & $\mathrm{C} 31-\mathrm{O} 4-\mathrm{C} 32-\mathrm{C} 33$ & $-165.3(3)$ \\
\hline $\mathrm{C} 5-\mathrm{C} 6-\mathrm{C} 7-\mathrm{C} 8$ & $0.8(6)$ & $\mathrm{C} 32-\mathrm{O} 4-\mathrm{C} 31-\mathrm{C} 30$ & $82.2(4)$ \\
\hline $\mathrm{C} 6-\mathrm{C} 7-\mathrm{C} 8-\mathrm{C} 9$ & $-0.6(6)$ & $\mathrm{C} 33-\mathrm{O} 1-\mathrm{C} 26-\mathrm{C} 27$ & $-166.2(3)$ \\
\hline $\mathrm{C} 7-\mathrm{C} 8-\mathrm{C} 9-\mathrm{C} 1$ & $-177.1(4)$ & $\mathrm{Li} 1-\mathrm{O} 1-\mathrm{C} 26-\mathrm{C} 27$ & $-42.0(4)$ \\
\hline $\mathrm{C} 7-\mathrm{C} 8-\mathrm{C} 9-\mathrm{C} 4$ & $-0.8(5)$ & $\mathrm{Li} 1-\mathrm{O} 1-\mathrm{C} 33-\mathrm{C} 32$ & $-35.0(4)$ \\
\hline $\mathrm{C} 9-\mathrm{C} 1-\mathrm{C} 2-\mathrm{C} 3$ & $-0.4(4)$ & $\mathrm{Li} 1-\mathrm{O} 2-\mathrm{C} 27-\mathrm{C} 26$ & $-38.1(4)$ \\
\hline $\mathrm{C} 9-\mathrm{C} 1-\mathrm{C} 10-\mathrm{C} 11$ & $-81.5(4)$ & $\mathrm{Li} 1-\mathrm{O} 2-\mathrm{C} 28-\mathrm{C} 29$ & $-45.6(4)$ \\
\hline $\mathrm{C} 9-\mathrm{C} 4-\mathrm{C} 5-\mathrm{C} 6$ & $-1.9(5)$ & $\mathrm{Li} 1-\mathrm{O} 3-\mathrm{C} 29-\mathrm{C} 28$ & $-35.1(4)$ \\
\hline $\mathrm{C} 10-\mathrm{C} 1-\mathrm{C} 2-\mathrm{C} 3$ & $176.5(3)$ & $\mathrm{Li} 1-\mathrm{O} 3-\mathrm{C} 30-\mathrm{C} 31$ & $-42.3(4)$ \\
\hline $\mathrm{C} 10-\mathrm{C} 1-\mathrm{C} 9-\mathrm{C} 4$ & $-176.4(3)$ & $\mathrm{Li} 1-\mathrm{O} 4-\mathrm{C} 31-\mathrm{C} 30$ & $-37.9(4)$ \\
\hline $\mathrm{C} 10-\mathrm{C} 1-\mathrm{C} 9-\mathrm{C} 8$ & $0.3(6)$ & $\mathrm{Li} 1-\mathrm{O} 4-\mathrm{C} 32-\mathrm{C} 33$ & $-44.7(4)$ \\
\hline $\mathrm{C} 11-\mathrm{N} 1-\mathrm{C} 12-\mathrm{C} 13$ & $166.3(3)$ & $\mathrm{Li} 1-\mathrm{N} 1-\mathrm{C} 11-\mathrm{C} 10$ & $171.8(3)$ \\
\hline $\mathrm{C} 12-\mathrm{N} 1-\mathrm{C} 11-\mathrm{C} 10$ & $-67.8(4)$ & $\mathrm{Li} 1-\mathrm{N} 1-\mathrm{C} 12-\mathrm{C} 13$ & $-80.7(3)$ \\
\hline
\end{tabular}

